\title{
Development of a validation instrument in myocardial perfusion imaging: results of first flow experiments
}

Marije E. Kamphuis, Gert Jan Pelgrim, Marcel J. W. Greuter, Riemer H. J. A. Slart, Cornelis H. Slump

Marije E. Kamphuis, Gert Jan Pelgrim, Marcel J. W. Greuter, Riemer H. J. A. Slart, Cornelis H. Slump, "Development of a validation instrument in myocardial perfusion imaging: results of first flow experiments," Proc. SPIE 10577, Medical Imaging 2018: Image Perception, Observer Performance, and Technology Assessment, 1057714 (7 March 2018); doi: 10.1117/12.2286337

SPIE. Event: SPIE Medical Imaging, 2018, Houston, Texas, United States 


\title{
Development of a validation instrument in myocardial perfusion imaging, results of first flow experiments
}

\author{
Marije E. Kamphuis*a , Gert Jan Pelgrim ${ }^{\mathrm{b}}$, Marcel J. W. Greuter ${ }^{\mathrm{ab}}$, Riemer H. J. A. Slart ${ }^{\mathrm{ab}}$, \\ Cornelis H. Slump ${ }^{\mathrm{a}}$ \\ ${ }^{a}$ MIRA Institute for Biomedical Technology and Technical Medicine, University of Twente, \\ Enschede, The Netherlands; ${ }^{b}$ Radiology \& Nuclear Medicine and Molecular Imaging Department, \\ University Medical Center Groningen, Groningen, The Netherlands
}

\begin{abstract}
Institutional diagnostic workflows regarding coronary artery disease (CAD) may differ greatly. Myocardial perfusion imaging (MPI) is a commonly used diagnostic method in CAD, whereby multiple modalities are deployed to assess relative or absolute myocardial blood flow (MBF) (e.g. with SPECT, PET, MR, CT, or combinations). In line with proper clinical decision-making, it is essential to assess institutional MPI test validity by confronting MBF assessment to a ground truth. Our research focuses on developing such validation instrument/method for MPI by means of simulating controlled myocardial perfusion in a phantom flow setup. A first step was made in the process of method development and validation by specifying basic requirements for the phantom flow setup. First tests in CT-MPI were aimed to gain experience in clinical testing, to verify to which extent the set requirements are met, and to evaluate the steps needed to further improve accuracy and reproducibility of measurements.

The myocardium was simulated as a static cylinder and placed in a controllable pulsatile flow circuit whereby using flow sensors as reference. First flow experiments were performed for different stroke volumes (20-35 mL/stroke). After contrast injection, dynamic MPI-CT scans (SOMATOM Force, Siemens) were obtained to investigate the relation between first-pass measured and computed flow. We observed a moderate correlation; hence, the required accuracy and reproducibility levels were not met. However, we have gained new insights in factors regarding the measurement setup and MBF computation process that might affect instrument validation, which we will incorporate in future flow setup design and testing.
\end{abstract}

Keywords: myocardial perfusion imaging, validation, CT, phantom, flow experiment

\section{INTRODUCTION}

Decision making in diagnosing coronary artery disease (CAD) involves a continuous trade-off between assessment of additional patient-specific information (e.g. clinical evaluation and testing), wait and see, or start intervention (e.g. prescribing medicine, surgery). Proper risk stratification and patient triage is thereby key, especially since the impact of 'wrong' diagnosis is high; it may lead to myocardial infarction, or even death. ${ }^{1,2}$ Death rates from cardiovascular disease, including CAD, have declined, yet the burden of disease remains high. ${ }^{2}$ International practice guidelines advise to start with anamnesis and physical examination to obtain a patient's pre-test probability, followed by basic testing such as ECG, rest echocardiography, and biochemical tests to rule out other disorders. ${ }^{3,4}$ If inconclusive, there are both anatomical and functional imaging methods to assess presence and severity of obstructive CAD. Examples of anatomical imaging methods include CT based coronary angiography (CA) and CA via catheterization. Such methods have a high negative predictive value. ${ }^{5,6}$ Nonetheless, stenosis severity is not always a good predictor for the presence of ischemia, in which functional imaging can play an important role. Examples of functional imaging methods include multimodal myocardial perfusion imaging (SPECT, PET, MR, CT, or combinations) and the determination of coronary or fractional flow reserve via catheterization. ${ }^{7-9}$

*m.e.kamphuis@utwente.nl; phone +31 (0)53 4892854

Medical Imaging 2018: Image Perception, Observer Performance, and Technology Assessment, edited by

Robert M. Nishikawa, Frank W. Samuelson, Proc. of SPIE Vol. 10577, 1057714

(C) 2018 SPIE · CCC code: 1605-7422/18/\$18 - doi: 10.1117/12.2286337 
Institutional diagnostic workflows regarding CAD may differ greatly. In this, hospitals have to make a trade-off between test performance, local expertise, resources and infrastructure. ${ }^{10}$ In order to make the right medical decision, it is important to identify institutional test performance, especially the grey zones that might influence medical decisionmaking. For example, current clinical routine in MPI involves visual evaluation of relative perfusion images, which could lead to false negative test results in patients with three vessel CAD. ${ }^{11}$ Evidence levels of MPI validation studies are reflected by its chosen reference standard. In a meta-analysis performed by Jaarsma et al. ${ }^{12}$, data from patient studies on test performance of different MPI methods were pooled using CA as reference standard. Up for discussion was the potential bias in their results as CA does indicate anatomical stenosis severity, but disregards its hemodynamic consequences. For these reasons, it is important to confront a test method to a 'ground truth'.

Quantitative analysis of MPI encompasses the assessment of absolute myocardial blood flow (MBF). These quantification techniques might contribute to the development of a validated reference in MPI, hence these techniques are widely recognized as research tool. ${ }^{13-14}$ However, such measures have not been adopted into clinical routine yet. One of the main reasons is the lack of standardization of the analysis methods, ${ }^{15}$ which is partly due to the lack of a gold standard for validation of the results. Novel techniques are currently developed using combinations of numerical simulations, animal studies and human trials. ${ }^{16,17}$ Our research focuses on developing a validation instrument/method for MPI. A first step was made in the process of method development and validation. We built a myocardial perfusion phantom in a controllable flow circuit and tested the setup in first-pass MPI-CT. In this way, we could verify to which extent the set requirements for instrument development were met so far.

\section{METHOD DEVELOPMENT AND VALIDATION}

\subsection{Approach}

The ultimate aim is to develop a validation instrument/method for MPI. Current research focuses on the first stage in method development and validation. This stage consists of three steps: (1) building a valid and controllable flow circuit simulating aortic and myocardial flow, (2) building a myocardial perfusion phantom, and (3) validating/verifying dynamic first-pass MPI-CT using the constructed measurement setup. The primary focus lies on building the flow circuit, which will shift in a later stage to improving myocardial perfusion simulation, and validation.

\subsection{Requirements}

Initial requirements to achieve valid and controllable flow are to control (a) myocardial perfusion rate (50-250 $\mathrm{mL} / 100 \mathrm{~g} / \mathrm{min}$ ), (b) cardiac output (pulsatile, stroke volume: 50-200 mL/stroke, heart rate: $45-150 \mathrm{bpm}$ ), and to measure (c) aortic and myocardial flow accurately (repeated measures (r.m.) ANOVA, p<0.05) and reproducibly (Pearson correlation coefficient $(\mathrm{PCC})>0.95, \mathrm{p}<0.05$ ). The set criteria represent the physiological range in patients with suspected $\mathrm{CAD}$ during stress and rest. Another requirement for the measurement setup incorporates easy replacement of the myocardial perfusion prototype phantom. This phantom requires CT compatible design of a static one-compartment volume that serves as cardiac insert in an anthropomorphic thorax phantom (for realistic $\mathrm{x}$-ray attenuation). Validation/verification of first-pass MPI-CT requires accurate (r.m. ANOVA, $\mathrm{p}<0.05$ ) and reproducible (PCC $>0.95$, $\mathrm{p}<0.05) \mathrm{MBF}$ computation for different perfusion rates and cardiac outputs using circuit measured myocardial flow as reference.

\section{MATERIALS AND METHODS}

\subsection{1 Materials}

The developed flow measurement setup (Figure 1) was designed to fit on a CT table and consisted of three main parts, namely (1) the pump and flow circuit, (3) the phantom, and (4) the control/measurement unit. 


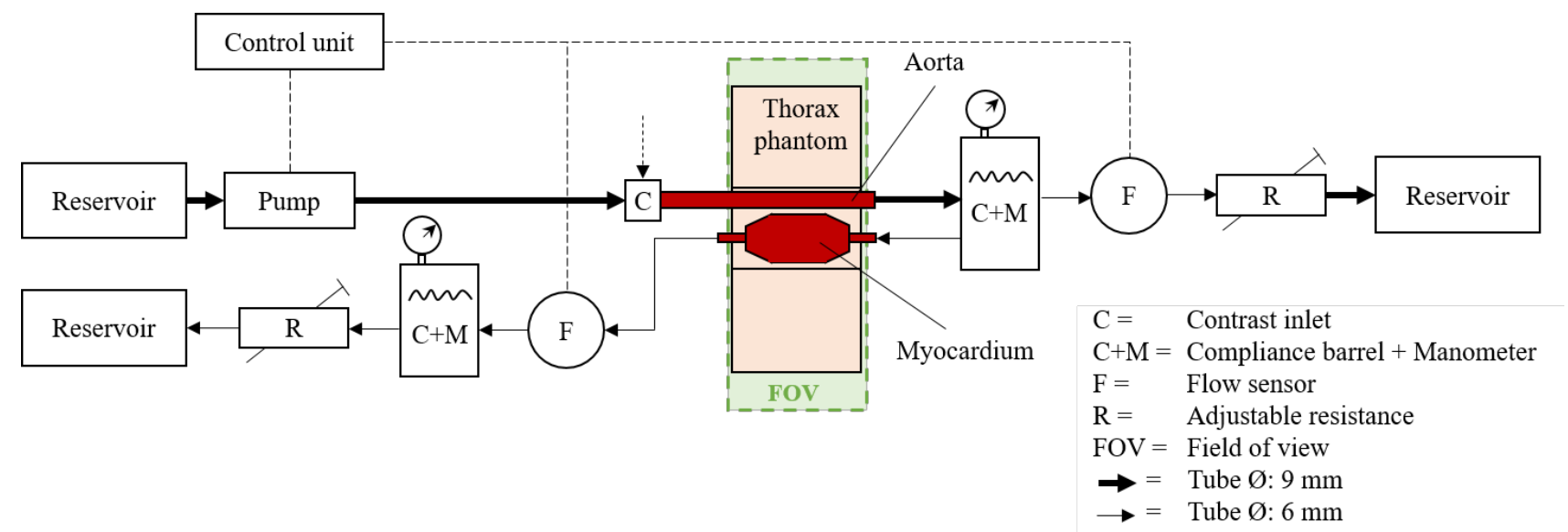

Figure 1. Flow measurement setup for first experiment in CT.

\section{The pump and flow circuit}

The flow measurement setup can be configured as an open or a closed circuit configuration. All the data presented in this paper were obtained with the open circuit setup to prevent contrast recirculation. The used piston pump (Vivitro Superpump, Vivitro Labs, Victoria, Canada) generates programmable pulsatile flow waveforms at variable amplitude and frequency. In this way, cardiac output in the ascending aorta can be simulated by adjusting the flow pattern, stroke volume and heartrate. Tap water is used for initial testing, though the pump is also compatible for other fluids (e.g. blood-mimicking fluids). Rigid, plastic tubes with an inner diameter of $9 \mathrm{~mm}$ were used to simulate the ascending and descending aorta as a straight line. A branch to the myocardium was made using a rigid plastic tube with an inner diameter of $6 \mathrm{~mm}$, roughly corresponding to the total inner diameter of the main coronary arteries combined. Compliance barrels and end resistances controlled systolic and diastolic blood pressures. A contrast insert was located $\sim 15 \mathrm{~cm}$ before entering the thorax phantom to mix inflowing water with the contrast agent on forehand.

\section{The phantom}

The left ventricle myocardium was simulated as a static, plastic one-compartment phantom (Figure 1) with a total volume, length and inner diameter of $170 \mathrm{~mL}, 9.0 \mathrm{~cm}$, and $3.5 \mathrm{~cm}$, respectively. Both the aorta and myocardial perfusion phantom were placed within an anthropomorphic thorax phantom (QRM, Möhrendorf, Germany). The scanner's Field of View (FOV) covered the thorax phantom.

\section{The control unit}

We used the ViVitest software (Vivitro Labs, Victoria, Canada) to control the cardiac output of the pump and to receive forward flow from the main pump and flow sensors in the flow circuit. The used flow sensors (Low-flow Flowmeters, FCH-mini-POM, Biotech, Germany) have +/- 0.5\% accuracy read out within a flow range of $0.05-3.0 \mathrm{~L} / \mathrm{min}$. Data acquisition took place before dynamic CT scanning for a period of 20 heart cycles.

\subsection{Methods}

\section{CT protocol}

First pass dynamic perfusion scans were acquired on a third generation dual source CT scanner (SOMATOM Force, Siemens Healthcare, Forchheim, Germany). The FOV was determined by a scout image. Data acquisition took place in conventional shuttle mode (z-range of $102 \mathrm{~mm}$ ), with electrocardiographic (ECG) triggering at end-systole, tube voltage of $80 \mathrm{kV}$ for both tubes, gantry rotation time of $280 \mathrm{~ms}$, and tube current of $450 \mathrm{mAs}$ per rotation. Scans were started $\sim 4$ seconds prior to contrast arrival in the aorta and had a total duration of 35 seconds. 
Dynamic CT-MPI data were reconstructed with $3.0 \mathrm{~mm}$ section thickness and $1.5 \mathrm{~mm}$ increment in the short-axis plane. Traditional filtered back projection was used with the "B23f" image reconstruction algorithm.

\section{Contrast administration}

The administration protocol was first optimized to acquire representative CT intensity peaks ( 350 HU) in the simulated aorta. For all dynamic CT-MPI scans, $15 \mathrm{~mL}$ of Iomeron350 was administered at an injection rate of $3 \mathrm{~mL} / \mathrm{s}$. The contrast to saline ratio was 60:40.

\section{MBF calculations}

MBF computations of the dynamic CT scans were executed by institutional software (Volume Perfusion CT myocardium software, MMWP VA41A, Siemens). A region of interest (ROI) was manually drawn in the aorta and myocardium. ROI selection in the myocardium was performed at the center of the myocardium in axial plane and covered the entire myocardial surface. The average intensity in the ROIs was subsequently plotted over time. Figure 2 shows an example of a typical TIC using CT-MPI for a healthy patient. The software computes the MBF in the selected myocardial region according to the upslope method:

$$
\text { MBF }=\text { MaxSlope }_{\mathrm{TIC}} / \text { MaxValue }_{\mathrm{AIF}}
$$

Whereby the MaxSlope ${ }_{\text {TIC }}$ stands for the maximum slope of the myocardial time intensity curve (TIC) and the MaxValue $_{\mathrm{AIF}}$ for the peak value of the arterial input function (AIF) curve measured in the aorta. The computed MBF is presented in $\mathrm{mL} / 100 \mathrm{~g} / \mathrm{min}$.

The computed MBF was verified using a flow sensor. The average measured flow was converted to real MBF by dividing it by the total volume of the myocardial perfusion phantom:

$$
\mathrm{MBF}=\text { AverageFlow }_{\text {sensor }} / \text { volume }_{\text {myo }}
$$

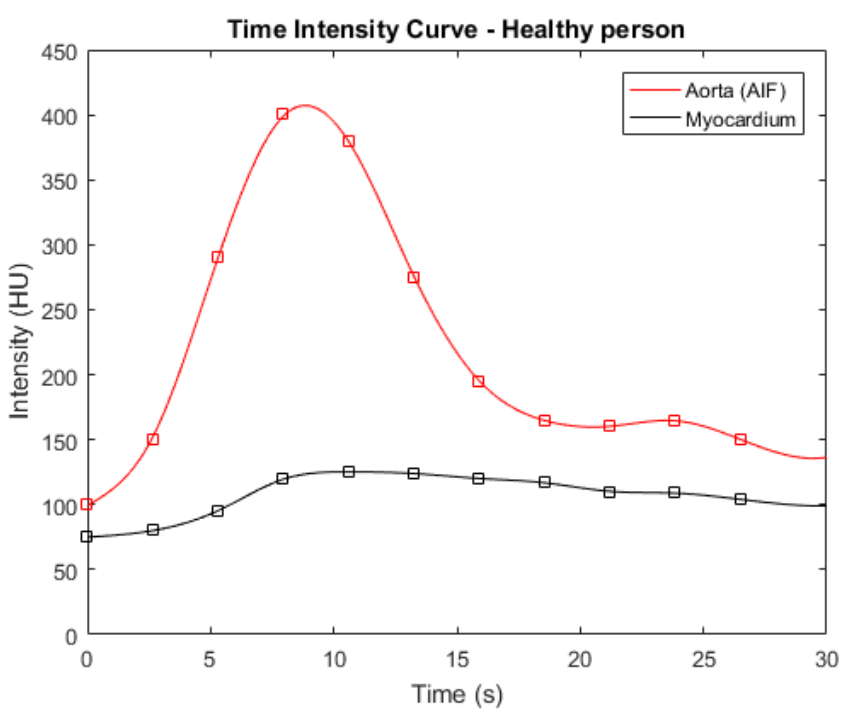

Figure 2. Example of a typical time intensity curve of the ascending aorta and myocardium in a healthy person. The markers over time indicate the amount of CT data acquired. Data were adapted from Pelgrim et al. ${ }^{18}$ 


\section{Sensitivity to cardiac output}

We performed data acquisition for varying stroke volumes $(20,25,30$, and $30 \mathrm{~mL} / \mathrm{stroke})$ to assess the effect of variations in cardiac output on the measured TICs and MBF computation. Other variables in the flows circuit were fixed.

\section{Reproducibility Experiments}

Reproducibility was assessed by repeating each measurement. At one specific stroke volume the measurement was repeated four times.

\section{Statistical analysis}

MBF computations and measurements were compared to assess reproducibility by means of a linear regression analysis using the Pearson's correlation coefficient. All data analysis was performed with Matlab (Mathworks, 2016a).

\section{PRELIMINARY RESULTS}

\subsection{Performance of flow sensors}

The response of the flow sensors is depicted for all measurements in Figure 3. Average deviation between pump generated cardiac output flow and measured flow was $0.1 \mathrm{~mL} / \mathrm{s}(\mathrm{SD}=0.06)$, indicating a highly accurate and reproducible ( $\mathrm{PCC}=1.00, \mathrm{p}<0.001$ ) flow acquisition. The flow ratio between aorta (after branch) and myocardium was 2.67:1 and remained constant for most measurements.

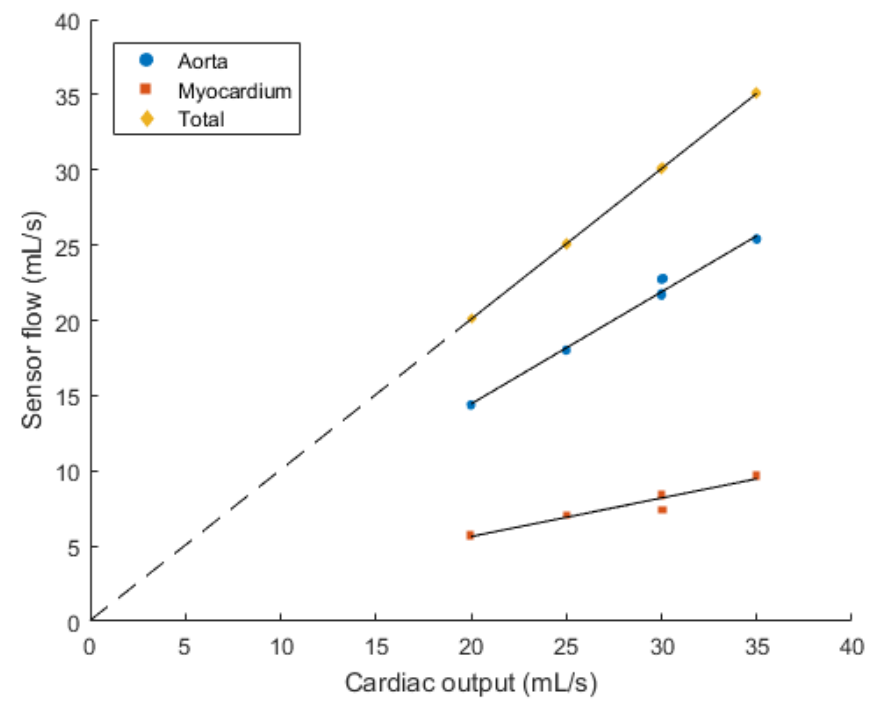

Figure 3. Response of the flow sensors along the simulated aorta and myocardium to different cardiac outputs. 


\subsection{Sensitivity and reproducibility to cardiac output}

Figure 4 shows the obtained TICs in the simulated aorta and myocardium for different cardiac outputs. Variation in peak intensity varies about $\sim 200 \mathrm{HU}$ and $\sim 50 \mathrm{HU}$ in the aorta and myocardium, respectively. In the myocardial TIC, an increase in upslope was visible for increased cardiac output between 30 and $35 \mathrm{~mL} / \mathrm{s}$, though the measurement at 25 $\mathrm{mL} / \mathrm{s}$ contained an even steeper upslope and higher maximum intensity, which was also the case for this measurement in the aortic TIC. In Figure 5, results from preliminary reproducibility measurements at a cardiac output of $30 \mathrm{~mL} / \mathrm{s}$ were presented. There is wide variation in obtained intensity peaks in the aorta and maximum upslope in the myocardium.

Figure 6 presents the relation between first-pass measured and computed flow for the first experiments in MPI-CT. No strong correlation between both methods was observed (PCC $=0.62, \mathrm{p}=0.03$ ).
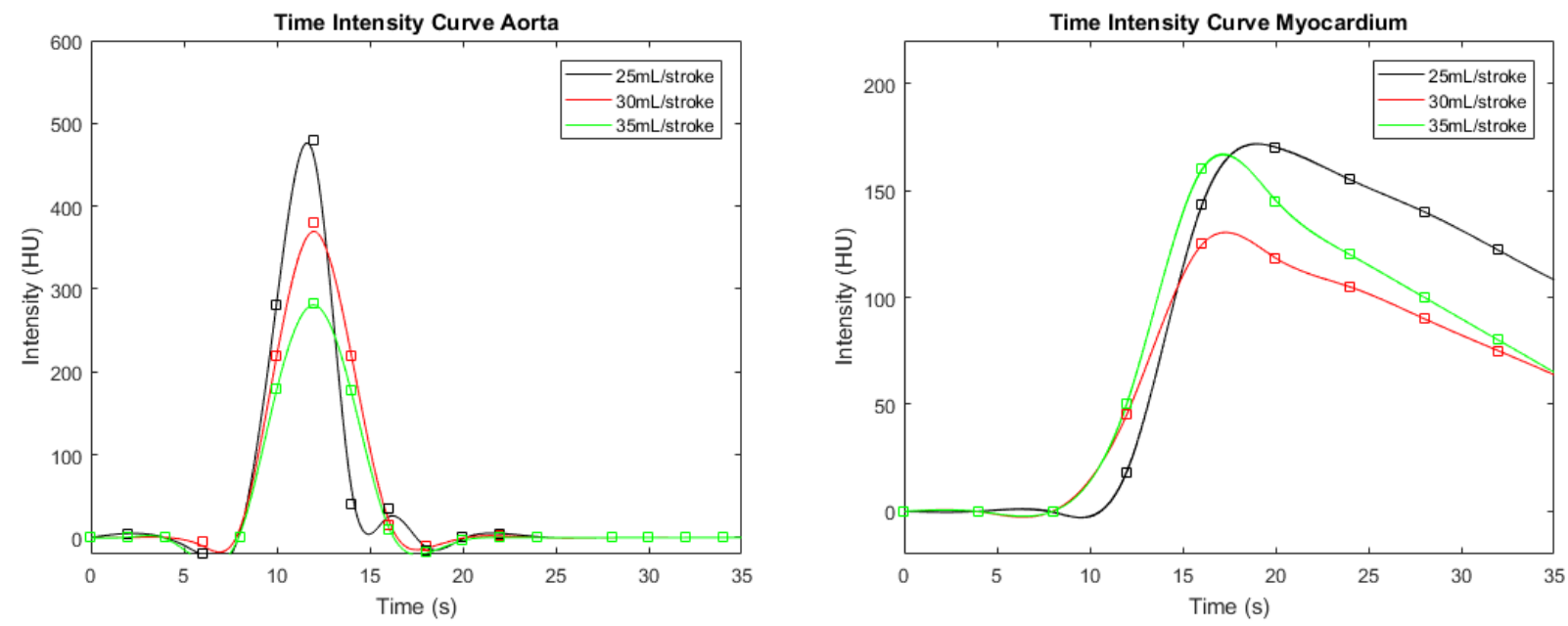

Figure 4. Sensitivity plot of time intensity curves for the simulated aorta and myocardium at different cardiac outputs.
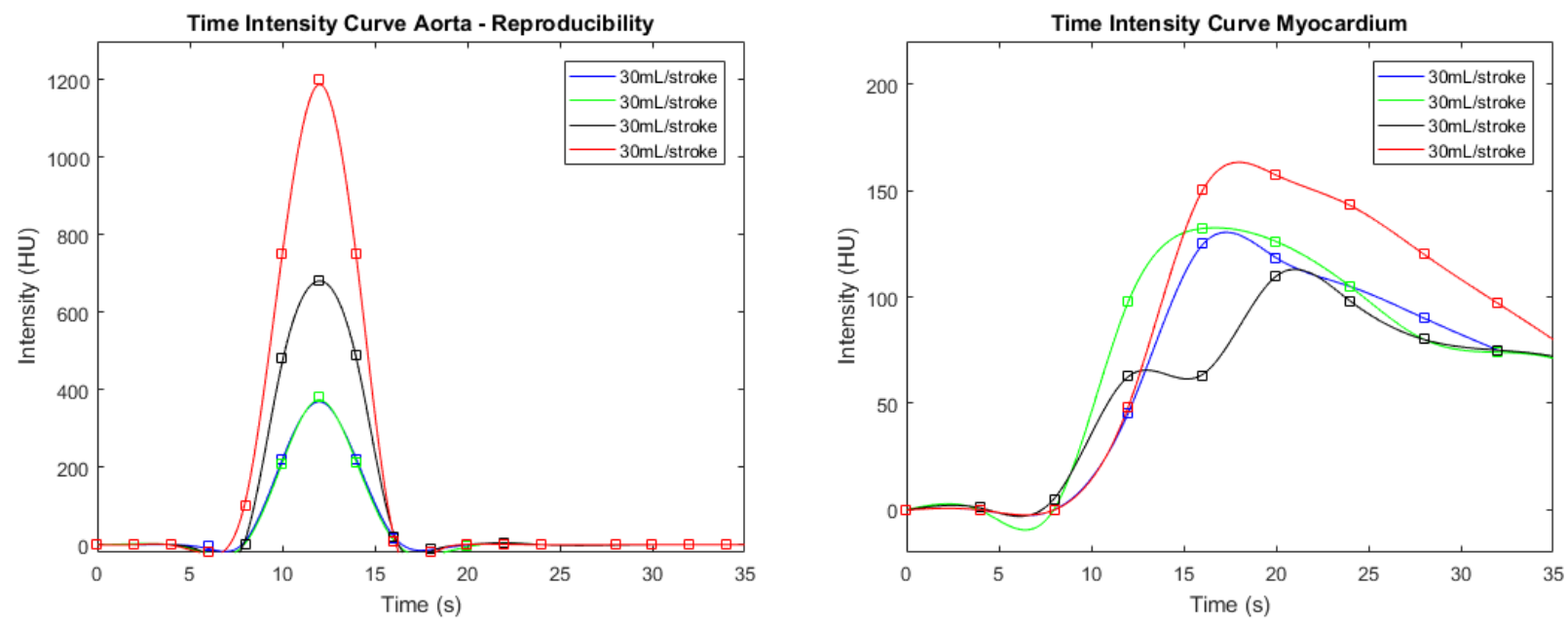

Figure 5. Reproducibility plot of time intensity curves for the simulated aorta and myocardium at a cardiac output of $30 \mathrm{~mL} / \mathrm{s}$ (Heart rate $=60 \mathrm{bpm})$. 


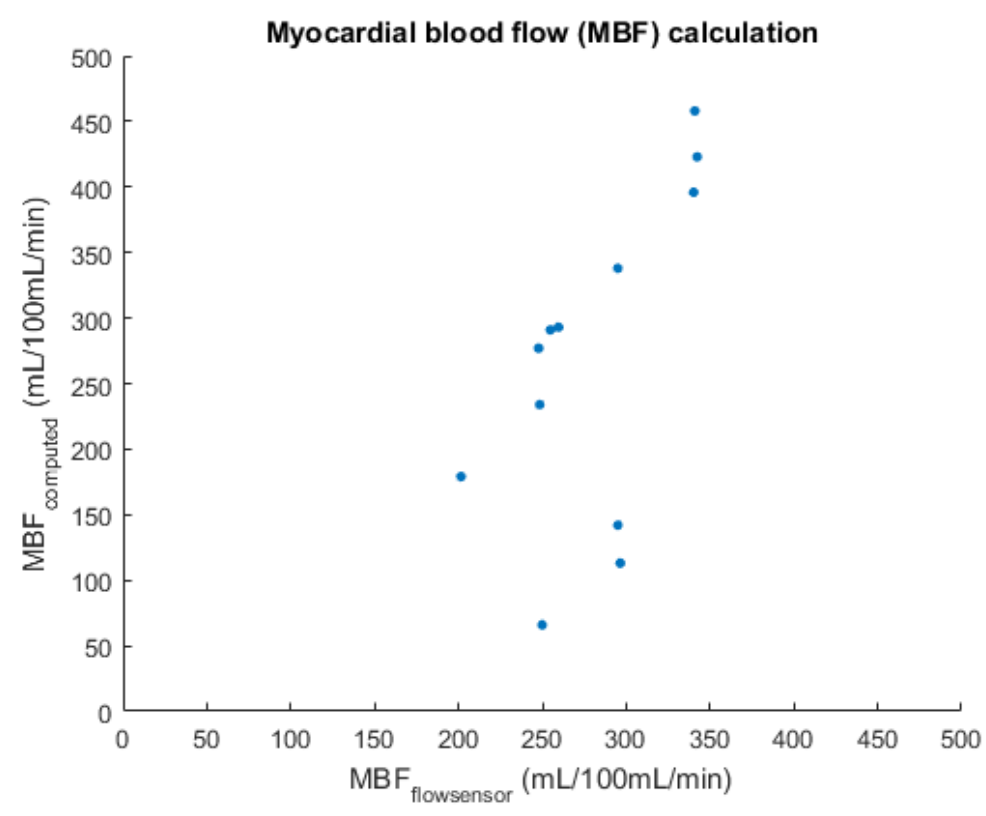

Figure 6. Preliminary results on myocardial blood flow calculation performed by the institutional software whereby measured flow by a flow sensor was used as reference.

\section{DISCUSSION}

Our ultimate aim is to develop a validation instrument for MPI. In this study, first steps in this development process were made by building a controllable flow circuit and conducting first-pass MPI flow experiments in CT. First stage requirements for the flow circuit were partly met, and for the myocardial perfusion phantom entirely. We can alter cardiac output and perfusion rates in the measurement setup by adjusting pump settings and tuning end resistances. Nevertheless, the setup is not validated for the set physiological range yet. Preliminary method validation experiments in CT showed a weak correlation between computed and measured MBF for different stroke volumes. Results were thereby not reproducible.

Underlying causes for the erroneous data can be associated to different processes:

- How well the flow circuit and myocardial perfusion phantom represents and measures relevant patient physiology,

- CT-MPI performance in the assessment of signal enhancement (i.e. myocardial flow), and

- Robustness and accuracy of data-analysis and MBF computation.

\subsection{Measurement setup and myocardial perfusion phantom}

From the data analysis we can conclude that the measured flows in aorta and myocardium add up to the generated cardiac output with good accuracy and reproducibility. There were no leakages in the circuit. However, the range in which results were obtained did not include the complete specified physiological range, which requires further validation. In addition, there were some design issues detected that need modification. Firstly, the simulated aorta had an inner diameter of $9 \mathrm{~mm}$, while a diameter $>20 \mathrm{~mm}$ is more realistic. However, surrounding connections with contrast inlet and compliance barrel were of smaller size. The reduced diameter had great impact on experimental design, as it altered the flow ratio between aorta and myocardium drastically. Tuning the end resistances could not negate this effect, 
resulting in a non-realistic flow ratio between simulated aorta and myocardium (see Figure 2 and 4). This flow ratio was also hampered by the inner diameter of the selected flow sensor in the aorta (see Figure 1), which served as an unwanted extra resistance. Thirdly, the used compliance barrels were not sufficient in damping pulsatile flows $>35 \mathrm{~mL} / \mathrm{s}$. The overall volume of the barrel was too small, causing air bubbles entering the circuit and jet formation that could damage the pressure sensors on top of the barrel. For that reason, stroke volumes were varied at a lower flow range compared to the required range. Adjustments to the flow circuit will be made accordingly. Nevertheless, these design issues in the measurement setup cannot clarify the obtained inaccuracy and poor reproducibility of the preliminary results.

The simulated myocardium consisted of a basic, static one-compartment, which caused inevitable turbulent flow patterns within the myocardium, which is good visible in the software display as visualized in Figure 7 . The focus of this study was not on phantom design. However, it becomes questionable whether the required accuracy and reproducibility levels for the measurement setup can be achieved using this simplified model. We hypothesize that because of the observed flow patterns in the simulated myocardium (including undesired and unpredictable back flow), the physiological contrast distribution might be affected. If that is the case, the derived TICs may become less robust and remain different in shape and size compared to human TICs. A future step is to test this hypothesis by computing the TIC for the entire myocardium instead of in one slice. In this way, we can see whether the average contrast enhancement is a more robust method for MBF computation or that phantom design requires further improvement.

\subsection{2 CT-MPI performance}

Another issue involves the shuttle mode of the CT scanner. Shuttle mode was part of the standard imaging protocol as the detector size is too small to image the human heart in one mode. The effect of back and forth movement on the water flow is unknown, though might affect test results, especially since there is no simulation of soft tissue. We expect this to contribute to unpredictability of the turbulent flow pattern in the myocardium. CT-MPI performance is moreover limited by the amount of dynamic scans acquired over time. In patient scanning, radiation exposure is an important factor to limit scan numbers. In addition, ECG triggering and shuttle mode limit the window of data acquisition. Nonetheless, the validation process of our measurement set up can be improved by excluding the shuttle mode and increasing the amount of dynamic scans.

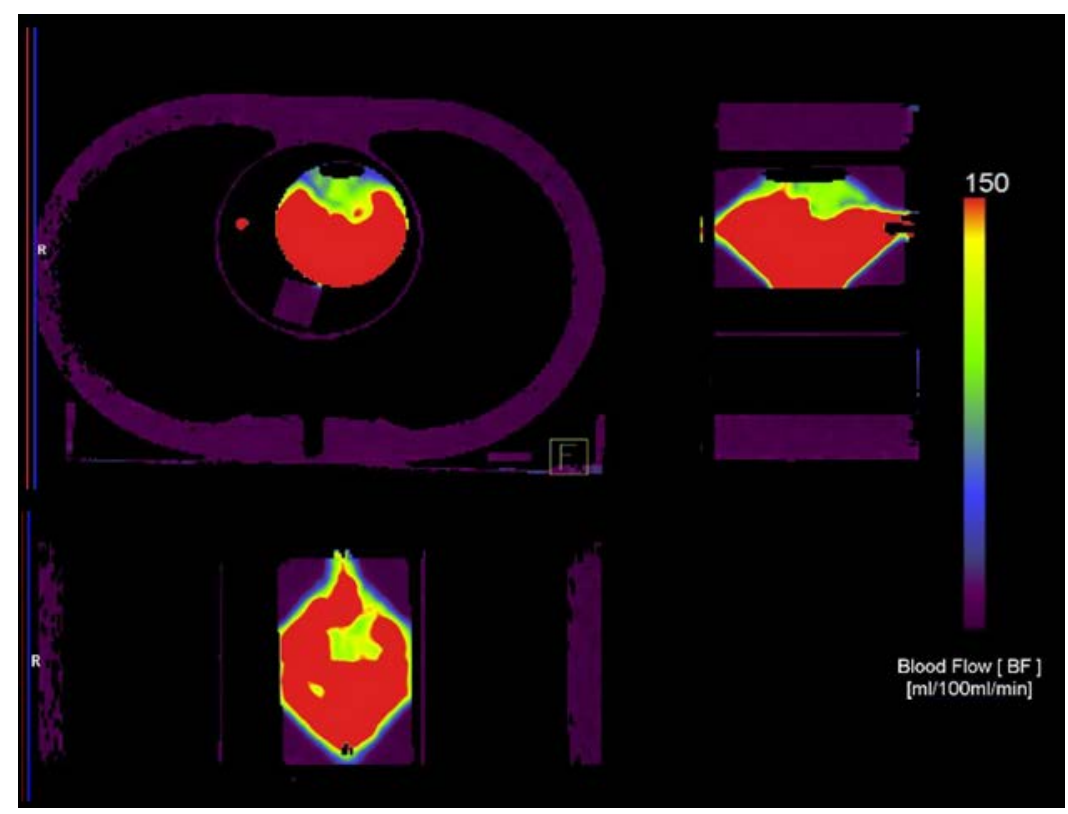

Figure 7. Axial, sagittal and coronal views of computed myocardial blood flow (MBF) using institutional software. Corresponding to a computed MBF of $66 \mathrm{~mL} / 100 \mathrm{~g} / \mathrm{min}$. Set pump flow: $25 \mathrm{~mL} / \mathrm{s}$, measured sensor MBF $250 \mathrm{~mL} / 100 \mathrm{~g} / \mathrm{min}$ ). 


\section{CONCLUSION}

The ultimate aim is to develop a validation instrument for MPI. This study focused on building a controllable measurement setup and gaining experience in MBF assessment in a clinical MPI-CT setting. We defined requirements for this first stage of method development, which were partially met. The myocardium was simulated as a static cylinder and placed in a controllable pulsatile flow circuit whereby using flow sensors as reference. First flow experiments were performed for different stroke volumes (20-35 mL/stroke). Dynamic contrast enhanced MPI-CT scans were obtained to investigate the relation between first-pass measured and computed flow. The required accuracy and reproducibility levels were not achieved (yet). Overall, we have gained general experience in clinical CT-MPI testing, which provided us with valuable new insights on future instrument design (for both the flow circuit and myocardial perfusion phantom), and on making the MBF quantification process more robust.

\section{REFERENCES}

[1] Yusuf, S.F.R.C.P., Reddy, S., Ôunpuu, S. and Anand, S.F.R.C.P, “Global burden of cardiovascular diseases”, Circulation, 104, 2855-2865 (2001).

[2] Heller, G.V. and Hendel, R.C., [Nuclear Cardiology: Practical Applications], McGraw-Hill Education.।, New York,223-244 (2011).

[3] Montalescot, G., Sechtem, U., Achenback, S., Andreotti, F., et al., "2013 ESC guidelines on the management of stable coronary artery disease,” Eur. Soc. of Cardiology 34, 2949-3003 (2013).

[4] Levine, G.N., Bates, E.R. Bittl, J.A., Brindis, R.G., Fihn, S.D., Fleisher, L.A., ... Smith, S.C., "ACC/AHA Focused update 2016 on duration of dual antiplatelet therapy in patients with coronary artery disease," Circulation 134, 134-155 (2016).

[5] Mowatt, G., Cummins, E., Waugh, N., Walker, S., Cook, J., Jia, X., et al., "Systemiatic review of the clinical effectiveness and cost-effectiveness of 64-slice or higher computed tomography angiography as an alternative to invasive coronary angiography in the investigation of coronary artery disease,” Health Tech. Assess. 12(17), iii,iv,ix-143 (2008).

[6] Paech, D.C., Weston, A.R., "A systematic review of the clinical effectiveness of 64-slice or higher computed tomography as an alternative to invasive coronary angiography in the investigation of suspected coronary artery disease,” BMC Cardiovasc Disord. 11-32 (2011).

[7] Holder, L.D.O., Lewis, S., Abrames, E., Wolin, E.A., “Review of SPECT myocardial perfusion imaging,” J Am Osteopath Coll Radiol., 5(3), 5-13 (2016).

[8] Schwitter, J., Nanz, D., Kneifel, S., Bertschinger, K., Büchi, M., Knüsel, P.R., Marincek, B., Lüscher, T.F., von Schulthess, G.K., "Assessment of myocardial perfusion in coronary artery disease by magnetic resonance", Circulation, 103, 2230-2235 (2001).

[9] Caruso, D., Eid, M., Schoepf, U.J., Nam Jin, K., Varga-Szemes, A., Tesche, C., Mangold, S., Spandorfer, A., Laghi, A., De Cecco, C.N., "Dynamic CT myocardial perfusion imaging," European Journal of Radiology, 85(10), 1893-1899 (2016).

[10] Roffi, M., Patrono, C., Collet, J.P., Mueller, C., Valgimigli, M., Andreotti, F., et al., "2015 ESC Guidelines for the management of acute coronary syndromes in patients presenting without persistent ST-segment elevation: Task Force for the Management of Acute Coronary Syndromes in Patients Presenting without Persistent STSegment Elevation of the European Society of Cardiology,” Eur Heart J., 37(3), 267-315 (2016).

[11] Mahajan, N., Polavaram, L., Vankayala, H. et al., "Diagnostic accuracy of myocardial perfusion imaging and stress echocardiography for the diagnosis of left main and triple vessel coronary artery disease: a comparative meta-analysis," Heart, 96, 956-966 (2010).

[12] Jaarsma., C. Leiner, T., Bekkers, S.C>, Crijns, H.J., Wildberger, J.E., Nagel, E., et al., "Diagnostic performance of noninvasive myocardial perfusion imaging using single-photon emission computed tomography, cardiac magnetic resonance, and positron emission tomography imaging for the detection of obstructive coronary artery disease: a meta-analysis,” J. Am Coll Cardiol, 59(19), 1719-28, (2012).

[13] Lee, D.C., Johnson, N.P., "Quantification of absolute myocardial blood flow by magnetic resonance perfusion imaging”, JACC Cardiovasc Imaging, 2(6), 761-70 (2009). 
[14] Hacker, M., “Absolute quantification of myocardial perfusion”, Circulation: Cardiovascular Imaging, 4,607-609 (2011).

[15] Morton, G.M.A., Chiribiri, A., Ishida, M., Hussain, S.T., Schuster, A., Indermuehle, A., Perera, D., Knuuti, J., Baker, S. Hedström, E., Schleyer, P., O’Doherty, M., Barrington, S., Nagel, E., “Quantification of absolute myocardial perfusion in patients with coronary artery disease," Journal of the American College of Cardiology, 60(16), 1546-55 (2012).

[16] Chiribiri, A., Schuster, A., Ishida, M., Hautvast, G., Zarinabad, N., Morton, G., Otton, J., Plein, S., Breeuwer, M., Batchelor, P., Schaeffter, T., Nagel, E., "Perfusion Phantom: an efficient and reproducible method to simulate myocardial first-pass perfusion measurements with cardiovascular magnetic resonance," Magnetic Resonance in Medicine, 69, 698-707 (2013).

[17] Pelgrim, G.J., Nieuwenhuis, E., Duguay, T.M., van der Geest, R., Varga-Szemes, A., Slump, C.H., Fuller, S.R., Oudkerk, M., Schoepf, U.J., Vliegenthart, R., "Optimal timing of image acquisition for arterial first pass CT myocardial perfusion imaging,” European Journal of Radiology, 86, 227-233 (2016). 